\title{
Distribuição do Profissional Médico na Macrorregião Norte do Paraná: Inequidade entre os Municípios de Diferentes Portes Populacionais
}

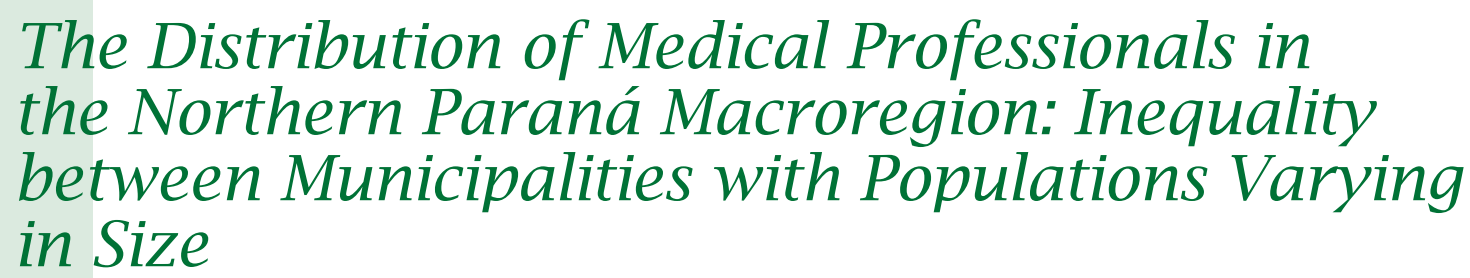

\section{PALAVRAS-CHAVE}

- Serviços Básicos de Saúde.

- Atenção Primária à Saúde.

- Recursos Humanos.

- Médicos.
Recebido em: 18/12/2016

Aprovado em: 04/01/2017
Carolina Milena Domingos ${ }^{I}$

Brígida Gimenez Carvalho ${ }^{I}$

William Augusto da Fonseca ${ }^{I}$

Luiz Cordoni JuniorI

Sônia Cristina Stefano Nicoletto ${ }^{I}$

RESUMO

A inadequada distribuição do profissional médico nas diferentes localidades do território nacional ocorre de várias formas: entre as regiões do País; entre capital e interior; entre áreas urbanas e rurais; entre áreas centrais e periféricas das regiões metropolitanas e entre assistência pública e privada. Desse modo, mesmo que se apresente uma relação medico/habitante adequada, a distribuição desses profissionais tende a se concentrar em determinadas áreas. Com o objetivo de analisar a distribuição dos médicos nos 97 municípios que compõem a macrorregião norte do Paraná, realizou-se estudo exploratório, descritivo,com base em dados secundários - Instituto Brasileiro de Geografia e Estatística (IBGE) e Sistema de Cadastro Nacional de Estabelecimentos de Saúde(SCNES)-acessados em outubro e novembro de 2014. Os resultados indicam uma distribuição desigual da população e dos médicos entre os 97 municípios da macrorregião. Os três municípios maiores abrangem 39,3\% da população e $56,1 \%$ dos médicos, enquanto nos outros 94 municípios em que residem 60,7\% da população atuam $43,9 \%$ dos médicos. Porém, a distribuição dos profissionais que fazem parte do Programa Mais Médicos ocorre de forma inversa, ou seja, metade desses médicos atua nos pequenos municípios em que reside aproximadamente um terço da população, o que demonstra que a distribuição destes profissionais contribui para melhorar o índice de médicos nos pequenos municípios. O enfrentamento das desigualdades na distribuição dos médicos nas diferentes regiões do País é complexo, não havendo soluções únicas e isoladas, sendo necessárias intervenções que articulem a gestão federal, estadual e municipal, estratégias de financiamento, formação dos profissionais de saúde e a regulação da formação e do processo de trabalho em saúde, políticas que fortaleçam as vulnerabilidades dos municípios menores. Investir na redução dessa desigualdade torna-se fundamental para a garantia da integralidade da atenção, sobretudo em localidades de difícil acesso, para assegurar o direito à saúde, especialmente em relação à carência de profissionais no âmbito do SUS. 


\section{KEYWORDS}

- Basic Health Services.

- Primary Healthcare.

- Human Resources.

- Physicians.
ABSTRACT

Inappropriate distribution of medical professional in different areas of the country occurs in several ways: between the regions of the country; between capital and countryside; between urban and rural areas; between central and peripheral areas of metropolitan regions and between public and private assistance. Thus, even if you submit a medical/proper inhabitant ratio, the distribution of these professionals tends to concentrate in certain areas. In order to analyze the distribution of physicians in the 97 municipalities that compose the Northern Parana Macroregion, held exploratory, descriptive study based on secondary data (Brazilian Institute of Geography and Statistics - IBGE and National Registration System of Health Establishments - SCNES), accessed in October and November 2014. The results indicate an unequal distribution of population and physicians among the 97 municipalities of the macro-region. The three largest municipalities cover $39.3 \%$ of the population and $56.1 \%$ of physicians while in the other 94 municipalities in which they reside $60.7 \%$ of the population, $43.9 \%$ of physicians work. However, when analyzing the distribution of professionals who are part of the more medical program, its distribution occurs in reverse, because half of these physicians work in small municipalities in which resides about a third of the population. These shows that the distribution of these professionals contributes to improving the health index in small municipalities. Addressing inequalities in the distribution of physicians in different regions of the country is complex, with no unique and isolated solutions, requiring interventions that coordinate federal, state and municipal management, financing strategies, training of health professionals and the regulation of health work process, policies that strengthen the vulnerabilities of smaller municipalities. Investing in reducing this inequality is fundamental to ensuring comprehensive care, especially in hard to reach locations, to ensure the right to health, especially in relation to the lack of professionals in the SUS.

\section{INTRODUÇÃO}

A inadequada distribuição de profissionais de saúde nas diferentes localidades do território nacional tem sido percebida ao longo dos anos, principalmente em relação ao profissional médico. Esta má distribuição ocorre de várias formas, podendo se apresentar entre as regiões do País; entre capital e interior; entre áreas urbanas e rurais; entre áreas centrais e periféricas das regiões metropolitanas e entre assistência pública e privada ${ }^{1}$. Desse modo, mesmo que se apresente uma relação medico/ habitante adequada, a distribuição desses profissionais tende a se concentrar em determinadas áreas ${ }^{2}$.Esta é uma realidade que também pode ser encontrada em outros países, como a Austrália (que chega a ter 0,9 médico por mil habitantes em suas áreas rurais) e os Estados Unidos, mas principalmente em regiões com situação econômica desfavorável, como países da África e do Sudoeste da Ásia ${ }^{3,4}$.

A quantificação do problema da distribuição médica no Brasil foi evidenciada na pesquisa "Demografia Médica do Brasil", publicada em 2015. Esse estudo aponta que o crescimento de médicos no País aumentou em maior velocidade do que o crescimento populacional. De 1970 (quando havia 58.994 registros) até 2015, o aumento foi de 633\%, enquanto a população brasileira cresceu 116\% no mesmo período. Em2015, o Brasil contava com uma população de 204.411.281 habitantes e com 399.692 médicos, o que significa um índice de1,95 médicos por mil habitantes ${ }^{5}$.

Ainda de acordo com esse estudo, as regiões Sudeste $(2,75)$ e Sul $(2,18)$ apresentaram os maiores índices de médicos por mil habitantes. Em contrapartida, as regiões Norte $(1,09)$ e Nordeste $(1,3)$ apresentaram as menores taxas, menos da metade das outras regiões.Ou seja, nas regiões onde está o maior número de pequenos municípios, há proporcionalmente o menor número de médicos. Quando se faz essa comparação por estado, a diferença aumenta, sendo o Distrito Federal o que apresentou a maior razão de médicos por mil habitantes do País $(4,28)$, seguido por Rio de Janeiro $(3,75)$ e São Paulo $(2,7)$. “No outro extremo, estão estados do Norte e Nordeste com menos de 1,6 médicos por mil habitantes", sendo o Maranhão o estado que apresentou o menor índice $(0,79)^{5}$.

Já na década de 1990, Machado ${ }^{6}$ constatou esse padrão ao identificar que quase $80 \%$ dos médicos estavam em apenas sete estados: Rio de Janeiro, São Paulo, Minas Gerais, Bahia, Pernambuco, Paraná e Rio Grande do Sul. Na época do estudo, esses eram os estados com maior concentração de renda e distribuição desigual dessa população, com cerca de $60 \%$ do contingente de médicos atuando nas capitais.Nos últimos anos, identifica-se que mais de $20 \%$ dos municípios do Brasil apresentam algum grau de escassez de médicos ${ }^{7}$. 
Os determinantes do problema da concentração de médicos no País são complexos, podendo ser citados: concentração de renda nas regiões Sul e Sudeste; direcionamento do mercado no exercício profissional; desigual distribuição de serviços de saúde; concentração da oferta de graduação e residência médica;precarização do trabalho no Norte e Nordeste, e investimento público insuficiente nessas regiões, levando a piores condições de trabalho e qualidade de vida ${ }^{1}$. Este cenário demonstra que a demografia médica no Brasil é um processo dinâmico, tensionado pelo comportamento e pelas escolhas profissionais, pelo mercado e por interesses econômicos, pelas resistências corporativas e pela regulação estatal sobre a formação médica e a profissão ${ }^{5}$.

Com o intuito de suprir a falta de profissionais em determinadas localidades do País, o governo anuncia a contratação de médicos estrangeiros para atuar em regiões onde as vagas ofertadas não sejam preenchidas por brasileiros. Também propõe a contratação temporária para regiões com quantidade insuficiente destes profissionais. E, ainda, anuncia medidas voltadas para o aumento de vagas nos cursos de Medicina e de residência médica, e realização de treinamento em serviços de atenção básica desde a graduação até a residência ${ }^{8}$.

Desde 1976, com o Programa de Interiorização das Ações de Saúde e Saneamento (Piass), já se começava a buscar formas de tentar fixar profissionais nas regiões, seguindo-se com o Programa de Interiorização do Sistema Único de Saúde (Pisus) em 1993 e o Programa de Interiorização do Trabalho em Saúde (Pits) em 2001. Como ações recentes do governo federal, destacam-se o Programa de Valorização dos Profissionais da Atenção Básica (Provab) e o Programa Mais Médicos (PMM)em 20133,9,10.

Em contrapartida,o Conselho Federal de Medicina (CFM) defende que as ações citadas não solucionarão o problema da distribuição de médicos, pois falta uma política de pessoal que estabeleça um plano de carreira atrativo para o serviço público. Também defende que a contratação de estrangeiros sem a devida avaliação do conhecimento destes profissionais poderia comprometer a qualidade do cuidado prestado à população ${ }^{8}$. Na opinião do CFM, caminha-se cada vez mais para a existência de alto número de médicos e simultânea carência destes profissionais em determinados locais, pois o aumento da quantidade destes não significará a sua disponibilidade para as regiões que mais necessitam, e a concentração continuará ocorrendo em estados com maior poder econômico ${ }^{5}$.

Para identificar tal carência nas regiões menos desenvolvidas, com maior dificuldade para fixar estes profissionais, é necessário fazer a análise no âmbito municipal. A relação entre médico e habitantes por região ou estado não consegue evidenciar as dificuldades dos municípios mais distantes, princi- palmente em relação à contratação e/ou fixação desse profissional, o que dificulta o acesso da população aos serviços de saúde e a continuidade da atenção.

A escolha da macrorregião norte do Paraná para realizar este estudo se deveu ao fato de estar sendo desenvolvida, nessa região, pesquisa sobre a gestão do trabalho nos municípios de pequeno porte (MPP), tendo sido verificada entre os resultados preliminares da pesquisa a dificuldade de contratação e de fixação de profissionais médicos. Desta forma, este estudo objetivou analisar a distribuição dos médicos nos 97 municípios que compõem a macrorregião norte do Paraná.

\section{METODOLOGIA}

Trata-se de um estudo exploratório, descritivo,que utilizou dados disponíveis eletronicamente nos bancos do Instituto Brasileiro de Geografia e Estatística (IBGE) ${ }^{11}$ e Sistema de Cadastro Nacional de Estabelecimentos de Saúde (SCNES) ${ }^{12}$, acessados em outubro e novembro de 2014, sendo os dados populacionais estimativas para 2015 referentes ao censo de 2010.

Desses sistemas foram obtidas informações sobre a classificação dos municípios por porte populacional, número de médicos por mil habitantes e número de médicos do Programa Mais Médicos.

Reconhece-se a limitação da base de dados secundária, pois a qualidade de suas informações depende da alimentação e atualização pelos responsáveis por inserir as informações. Apesar da obrigatoriedade de os serviços de saúde (públicos e privados) e os profissionais que atuam nesses serviços estarem cadastrados no SCNES, esses dados cadastrais nem sempre são mantidos atualizados, especialmente em serviços privados. Uma eventual desatualização dos dados cadastrais em serviços públicos é menos provável na atualidade, pois os serviços são constantemente monitorados quanto à sua atualização, tendo em vista que o repasse, regular e automático, dos recursos do Sistema Único de Saúde (SUS) do nível nacional para os municípios está condicionado a essa atualização ${ }^{13}$.

Desta forma, acredita-se que, como a maioria dos $97 \mathrm{mu}$ nicípios em que se realizou a pesquisa é de pequeno porte (83\%), com serviços quase exclusivamente públicos, a possível desatualização dos dados cadastrais não alteraria de forma significativa os resultados apresentados neste estudo.

Fizeram parte do estudo os cadastros de profissionais médicos dos 97 municípios da macrorregião norte do Paraná,

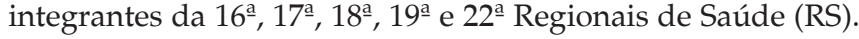
Os municípios foram categorizados segundo o porte populacional em municípios de pequeno porte 1 (população de até 20 mil habitantes); municípios de pequeno porte 2 (população de 20.001 a 50 mil habitantes);municípios de médio porte (popu- 
lação entre 50.001 e 100 mil habitantes); municípios de grande porte (população de 101 mil habitantes até 900 mil habitantes) e metrópoles (com mais de 900 mil habitantes) ${ }^{14}$. Os dados foram coletados por meio de uma ficha padronizada pelos autores, e para a análise utilizou-se o Microsoft Office Excel2010.

Este estudo integrou uma pesquisa maior, denominada "O jogo social nas Comissões Intergestores Regionais do norte do Paraná: a gestão compartilhada", aprovada e financiada pelo CNPq.A pesquisa foi norteada pelos princípios éticos contemplados na resolução $n^{\circ} 466 / 12$ do Conselho Nacional de Saúde. O projeto foi aprovado pelo Comitê de Ética em Pesquisa Envolvendo Seres Humanos da Universidade Estadual de Londrina sob o n 278/2011, CAAE-0258.0.268.268-11.

\section{RESULTADOS E DISCUSSÃO}

Distribuição dos médicos na macrorregião norte do Paraná A Tabela 1 apresenta o número de médicos, incluindo os do Programa Mais Médicos, nos municípios de diferentes portes das RS.Observa-se pouca variação no número de municípios que compõem as cinco regiões que integram a macrorregião norte do Paraná, sendo a $22^{\text {a }}$ aquela com menor número de municípios (16, o que correspondeu a $16,6 \%$ do total de municípios da região) e a 19a a que possui o maior número de municípios (22, o que correspondeu a $22,7 \%$ do total de municípios). No entanto, outras duas variáveis apresentadas nesta tabela (população e número de médicos) apresentaram resultados muito díspares quando comparados os municípios dessas regiões.

A $17^{a}$ RS é a região com maior contingente populacional (46,8\% da população) e também a que apresenta maior número de médicos (60,7\% do total), estando estes concentrados principalmente nos municípios de médio e grande porte (52,2\% do total).Esta regional apresenta maior número de estabelecimentos de saúde e maior concentração de serviços de média e alta complexidade ${ }^{15,16}$, ou seja, maior desenvolvimento socioeconômico e oferta de serviços ${ }^{17}$. Estas características

\begin{tabular}{|c|c|c|c|c|c|c|c|c|}
\hline \multicolumn{9}{|c|}{ 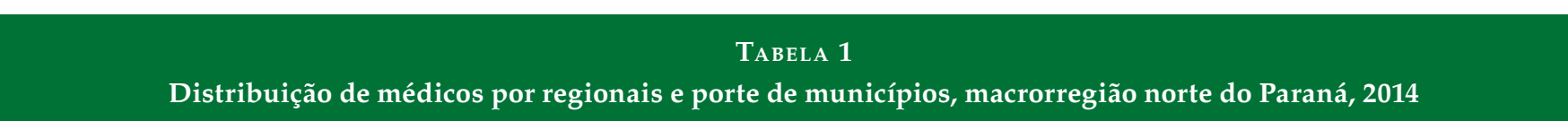 } \\
\hline & \multicolumn{2}{|c|}{ Municípios } & \multicolumn{2}{|c|}{ População } & \multicolumn{2}{|c|}{ Médicos } & \multicolumn{2}{|c|}{ Médicos do Mais Médicos } \\
\hline & $\mathbf{N}$ & $\%$ & $\mathbf{N}$ & $\%$ & $\mathbf{N}$ & $\%$ & $\mathbf{N}$ & $\%$ \\
\hline Total 16 RS & 17 & 17,5 & 346.972 & 18,6 & 727 & 17,1 & 27 & 22,5 \\
\hline Pequeno porte 1 & 14 & 14,4 & 101.634 & 5,4 & 126 & 3,0 & 11 & 9,2 \\
\hline Pequeno porte 2 & 1 & 1,0 & 20.269 & 1,1 & 48 & 1,2 & 0 & 0 \\
\hline Médio porte & 0 & 0 & 0 & 0 & 0 & 0 & 0 & 0 \\
\hline Grande porte & 2 & 2,1 & 225.069 & 12,1 & 553 & 12,9 & 16 & 13,3 \\
\hline Total 17 RS & 21 & 21,6 & 871.277 & 46,8 & 2.591 & 60,7 & 35 & 29,2 \\
\hline Pequeno porte 1 & 17 & 17,5 & 161.783 & 8,7 & 212 & 5,0 & 11 & 9,2 \\
\hline Pequeno porte 2 & 1 & 1,0 & 48.198 & 2,6 & 149 & 3,5 & 5 & 4,2 \\
\hline Médio porte & 2 & 2,1 & 154.595 & 8,3 & 386 & 9,0 & 5 & 4,2 \\
\hline Grande porte & 1 & 1,0 & 506.701 & 27,2 & 1.844 & 43,2 & 14 & 11,6 \\
\hline Total 18 RS & 21 & 21,6 & 226.234 & 12,2 & 380 & 8,9 & 13 & 10,8 \\
\hline Pequeno porte 1 & 18 & 18,6 & 126.512 & 6,8 & 131 & 3,1 & 10 & 8,3 \\
\hline Pequeno porte 2 & 3 & 3,0 & 99.722 & 5,4 & 249 & 5,8 & 3 & 2,5 \\
\hline Médio porte & 0 & 0 & 0 & 0 & 0 & 0 & 0 & 0 \\
\hline Grande porte & 0 & 0 & 0 & 0 & 0 & 0 & 0 & 0 \\
\hline Total 19 RS & 22 & 22,7 & 278.141 & 14,9 & 425 & 9,9 & 27 & 22,5 \\
\hline Pequeno porte 1 & 18 & 18,6 & 143.676 & 7,7 & 151 & 3,5 & 15 & 12,5 \\
\hline Pequeno porte 2 & 4 & 4,1 & 134.465 & 7,2 & 274 & 6,4 & 12 & 10 \\
\hline Médio porte & 0 & 0 & 0 & 0 & 0 & 0 & 0 & 0 \\
\hline Grande porte & 0 & 0 & 0 & 0 & 0 & 0 & 0 & 0 \\
\hline Total 22 RS & 16 & 16,6 & 138.389 & 7,5 & 144 & 3,4 & 18 & 15,0 \\
\hline Pequeno porte 1 & 15 & 15,6 & 106.573 & 5,7 & 75 & 1,8 & 13 & 10,8 \\
\hline Pequeno porte 2 & 1 & 1,0 & 31.816 & 1,8 & 69 & 1,6 & 5 & 4,2 \\
\hline Médio porte & 0 & 0 & 0 & 0 & 0 & 0 & 0 & 0 \\
\hline Grande porte & 0 & 0 & 0 & 0 & 0 & 0 & 0 & 0 \\
\hline Total & 97 & 100 & 186.1013 & 100 & 4.267 & 100 & 120 & 100 \\
\hline
\end{tabular}

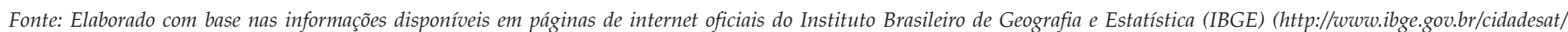
topwindow.htm?1) e Cadastro Nacional de Estabelecimentos de Saúde (CNES) (http://cnes.datasus.gov.br/), Brasil, 2014. 
podem justificar a maior quantidade de profissionais médicos presente nestes locais, pois é frequente a concentração desse profissional em grandes centros urbanos e atuando no meio hospitalar ${ }^{18}$.

Quanto à distribuição de médicos nos municípios da macrorregião, verifica-se que a maioria dos médicos (56,1\%) está concentrada nas três cidades de grande porte, que abrangem 39,3\% da população, enquanto nos outros 94 municípios, em que residem $60,7 \%$ da população, atuam $43,9 \%$ dos médicos. Esses resultados apontam uma distribuição desigual dos médicos na macrorregião norte do Paraná, semelhante ao que ocorre em outras regiões do País, em que estes profissionais se concentram nos grandes centros ${ }^{2,9}$.

Assim, quanto menor o município, menor também é a presença deste profissional, pois menores são os atrativos que influenciam a sua escolha pelo local de trabalho. Características desses municípios menores, como estar localizado em áreas mais isoladas e possuir maior vulnerabilidade econômica e social, podem ter contribuído para o menor contingente de médicos nesses locais ${ }^{7}$. Vale ressaltar que, do total de 97 municípios da macrorregião norte, $92(94,8 \%)$ são de pequeno porte 1 ou 2, e destes 82 municípios (84,5\%) são de pequeno porte 1 .

Também no País, quando se analisa a distribuição de médicos nos municípios por estrato populacional, verifica-se que nos 1.247 municípios com até 5 mil habitantes vivem 2,1\% da população do País e contam com $0,2 \%$ do conjunto dos médicos. Já nas 39 cidades com mais de 500 mil moradores, em que vivem $29,4 \%$ dos habitantes do País, atuam $60,9 \%$ de todos os médicos 5 .

O estudo de Girardi ${ }^{7}$ demonstrou que a escassez de médicos em municípios de pequeno porte 1 é uma realidade do território brasileiro, pois, dos 1.304 municípios brasileiros com algum grau de falta do profissional médico, $875(67,1 \%)$ eram municípios de pequeno porte 1. O relatório do Estudo Demografia Médica ${ }^{5}$ revelou que em municípios de até 5 mil habitantes a razão é de 0,23 médico por mil habitantes; nas localidades que têm de 5 a 10 mil habitantes, a razão é de 0,28 médico; e entre 10 mil e 20 mil, a razão é de 0,36 médico por mil habitantes.

Situação enfrentada pelos municípios e mais evidente para os municípios menores é a condição de gestores como entes federados autônomos(responsáveis pelas ações e serviços de saúde)adquirida pelo Estado brasileiro sem ter sido acompanhada por uma promoção de condições mínimas de infraestrutura econômica e social na maioria destes. Essa situação influenciou a capacidade de pagamento de salários, bem como a oferta de boas condições de trabalho e contribuiu para dificultar a fixação dos profissionais ${ }^{10}$.
Quanto à distribuição dos 120 médicos do PMM, verifica-se a presença desses profissionais nas cinco regiões e na maioria dos municípios da macrorregião, porém sua distribuição ocorre de forma inversa, ou seja, 50\% desses médicos estão lotados nos municípios de pequeno porte 1 , em que residem $34,3 \%$ da população. Dessa forma, a distribuição desses profissionais nessa macrorregião está contribuindo para melhorar o índice de médicos nos pequenos municípios.Pode-se afirmar que esse resultado mostra o atendimento a um dos objetivos do programa, que é o de levar mais médicos para regiões onde há escassez ou ausência desses profissionais ${ }^{3,19}$.

Como já afirmado, os municípios menores são aqueles que geralmente apresentam maior vulnerabilidade e tendem a ter maior escassez de médicos.Nesse sentido, uma política de provimento desses profissionais constitui uma possibilidade para suprir tal carência nessas localidades ${ }^{20}$. E, ainda,esses profissionais foram importantes para compor as equipes de saúde da família que se encontravam sem a participação do médico, o que fragilizava a integralidade do atendimento prestado por essas equipes, pois sem esse profissional sua atuação ficava limitada ${ }^{18}$.

Uma das dificuldades das equipes de saúde dos municípios menores se refere à alta rotatividade dos profissionais, principalmente do médico ${ }^{21,22}$. Entre as razões que favorecem essa rotatividade pode-se citar a forma de contratação (muitas vezes precária), o perfil desses profissionais (recém-formados que deixam as equipes de saúde da família para cursar a residência médica) e as condições de trabalho (pressão por produtividade de consultas e falta de capacitação e de retaguarda dos demais níveis de atenção) ${ }^{23}$.

Ney e Rodrigues ${ }^{24}$ apontam outros fatores que geram muitos problemas relacionados à permanência de médicos na estratégia saúde da família:inexistência de plano de cargos, carreiras e salários (PCCS), falta de estrutura para trabalhar, condições insalubres das unidades de saúde e ingerência política. Essa situação é mais acentuada em municípios de pequeno porte, que, muitas vezes, não conseguirem ter condições mínimas de infraestrutura, o que dificulta a fixação dos médicos nesses locais ${ }^{10}$. Assim, além do PMM, é preciso desenvolver outras ações para a fixação de médicos nos municípios menores. Uma das estratégias possíveis é a formulação de políticas públicas e de gestão macroeconômicas que promovam o desenvolvimento das regiões com maior vulnerabilidade (social, econômica ou de acesso) $)^{5}$.

A Organização Mundial da Saúde (OMS) e a Organização Pan-Americana da Saúde (Opas) não estabelecem taxas "ideais" de número de médicos por habitante, pois entendem queo estabelecimento desse índice depende de fatores regionais, 
Figura 1

Índice de médicos por mil habitantes segundo municípios das regionais de saúde da macrorregião norte do Paraná, 2014

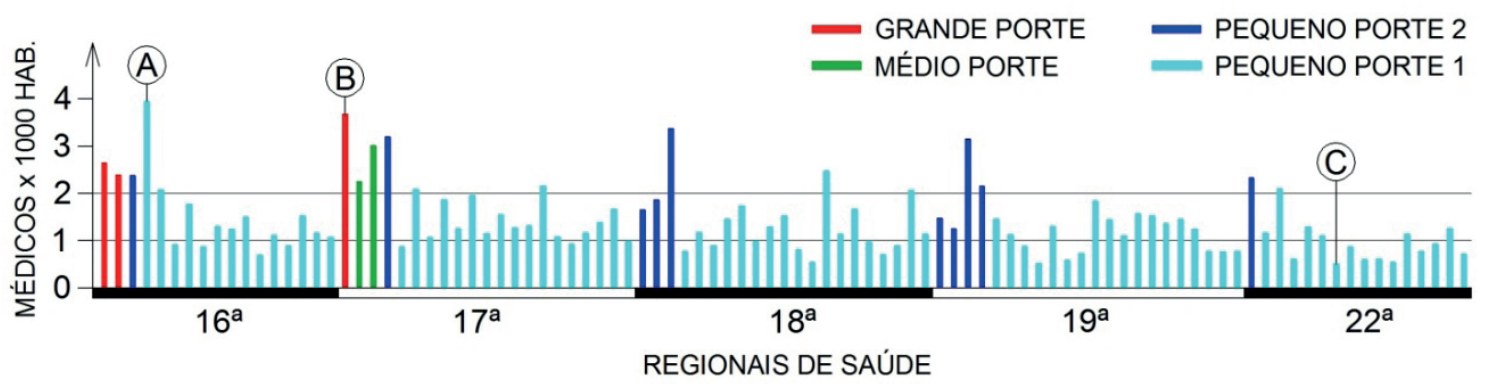

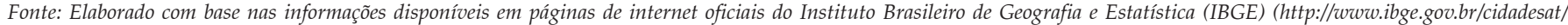
topwindow.htm?1) e Cadastro Nacional de Estabelecimentos de Saúde (CNES) (http://cnes.datasus.gov.br/), Brasil, 2014.

socioeconômicos, culturais e epidemiológicos 5 . Entretanto, é evidente que um número insuficiente de médicos se reflete diretamente na disponibilidade de assistência à saúde, em todos os níveis de atenção.

Nesse sentido, uma das formas de avaliar a disparidade entre a participação de cada estado no total da população e no total de médicos é calcular o número de médicos por mil habitantes. A Figura 1 apresenta o índice de médicos por mil habitantes, de acordo com o porte dos municípios,nas regionais de saúde da macrorregião norte. Verificou-se que $91,46 \%$ dos municípios de pequeno porte 1 apresentaram uma taxa de médicos inferior à média brasileira (1,95 médicos / mil habitantes), com destaque para Jardim Alegre, município $C$, pertencente à 22a RS (com taxa de 0,50 médico/mil habitantes), por possuir a menor taxa de médicos por mil habitantes da macrorregião norte.

Essa realidade é oposta à dos municípios de pequeno porte 2 , de médio porte e grande porte. Apenas quatro dos dez municípios de pequeno porte 2 apresentaram taxa abaixo da média nacional, sendo dois pertencentes à $18^{\mathrm{a}} \mathrm{RS}$ (Andirá e Bandeirantes, com índices de 1,65 e 1,86 médicos / mil habitantes, respectivamente) e dois pertencentes à 19 $9^{\mathrm{a}}$ RS (Cambará e Ibaiti, com índices de 1,47 e 1,25 médicos/mil habitantes, respectivamente). Foram identificados índices superiores a 2 médicos/mil habitantes em todos os municípios de médio e grande porte, com destaque para Londrina (município B, pertencente à $17^{\mathrm{a}} \mathrm{RS}, \mathrm{com}$ 3,67 médicos/mil habitantes).

Existe uma relação entre o número de médicos e o desenvolvimento econômico de uma região. Os municípios de maior porte do Paraná também estão entre as maiores economias do estado, pois têm maior representatividade no Produto Interno Bruto (PIB) do Paraná, com destaque para Londrina, que teve um PIB per capita de R\$29.63525.Assim, diferentes ganhos monetários entre as regiões influenciam a escolha do local de trabalho dos médicos, que são atraídos por ganhos mais elevados ${ }^{26}$.

O aumento do índice de médicos com o aumento da renda local, evidenciado por alguns estudos ${ }^{27,28,29}$, pode explicar o alto índice de médicos por mil habitantes (3,94 médicos / milhabitantes) apresentado pelo município de Sabáudia (município $\mathrm{A}$, pertencente à $16^{\mathrm{a}} \mathrm{RS}$ ). Esse município, apesar de ser de pequeno porte 1 , apresentou o maior índice de médicos por mil habitantes e o maior PIB per capitada macrorregião norte (R\$ 47.059) ${ }^{25}$.Outros municípios com o mesmo porte populacional de Sabáudia apresentaram menores PIB per capita ${ }^{25}$ que este município, como, por exemplo: Bom Sucesso (R\$14.540) e Grandes Rios (R\$13.449) na $16^{\mathrm{a}} \mathrm{RS} ; \mathrm{Guaraci}(\mathrm{R} \$ 15.364)$ na $17^{\mathrm{a}}$ RS; Sapopema (R\$ 10.943) na $18^{a}$ RS; Pinhalão (R\$ 17.474) na $19^{a}$ RS e Lunardelli (R\$ 11.887) na 22a RS, e todos apresentaram índices de médicos inferiores aos da média nacional.

O que se observa é que, mesmo em regiões com predominância de índice de médicos/mil habitantes próximo à média nacional, esses profissionais tendem a se concentrar em certos municípios, gerando um resultado socialmente indesejado, pois tal distribuição influencia o bem-estar social, uma vez que esses profissionais são provedores dos serviços de saúde ${ }^{2}$.

A $22^{\text {a }}$ regional de saúde possui os menores índices de médicos/mil habitantes da macrorregião norte, havendo predominância de menos de um médico/mil habitantes na maioria dos municípios que a compõem. Já a $17^{\text {a }}$ regional de saúde apresenta os maiores índices de médicos/mil habitantes da macrorregião; apenas três municípios possuem índices inferiores a um médico/mil habitantes, sendo predominante o índice maior que 1 e menor que 2 médicos $/$ mil habitantes. A 
$16^{\mathrm{a}}$ e a $17^{\mathrm{a}} \mathrm{RS}$ tiveram maior quantitativo de municípios com índices superiores a 2 médicos/mil habitantes. Destaca-se novamente a $17^{\mathrm{a}} \mathrm{RS}$, que, além das características atrativas para o médico já sugeridas,possui um município que oferta vagas em programas de residência médica, o que pode influenciar a escolha do local de trabalho pelo médico.

Póvoa e Andrade ${ }^{2}$, que descreveram e analisaram a distribuição geográfica e a decisão locacional dos médicos no Brasil, apresentaram,entre os seus resultados, que a oferta de vagas em programas de residência médica atrai médicos em busca de aperfeiçoamento, o que pode indicar a concentração dos programas de residência como um dos fatores que promovem a desigual distribuição geográfica dos médicos em diferentes regiões.Esse estudo também identificou uma atração exercida pelo fator econômico (estados com maior PIB per capita) e pelo papel do cônjuge (das oportunidades de mercado de trabalho disponíveis para o cônjuge)como fatores significativos na decisão locacional do médico.O complexo hospitalar pode ser outro fator atrativo para a carreira médica. Fleury e Ouverney ${ }^{30}$ percebem esta relação quando referem que tanto os hospitais como os profissionais de saúde estão concentrados nas regiões ricas do País.

Por fim, cabe considerar que a escolha do local para exercer a profissão também resulta de sua trajetória histórica quanto ao mercado de trabalho e formação médica. Estes, já pensados desde a década de 1960, vieram atender aos interesses privados, ao sistema de custeio, pagamento e lucratividade, aliados ao crescimento populacional, o que teve influência sobre a distribuição desses profissionais nas diversas regiões brasileiras ${ }^{10}$.

\section{CONSIDERAÇÕES FINAIS}

Este artigo procurou analisar a distribuição dos médicos nos municípios integrantes da macrorregião norte do Paraná. Os resultados indicamuma distribuição desigual da população e dos médicos entre os 97 municípios da macrorregião, sendo que apenas três municípios concentram pouco mais de um terço da população e mais da metade dos médicos. Porém,a distribuição dos profissionais que fazem parte do PMM ocorre de forma inversa, ou seja, metade desses médicos atua nos municípios de pequeno porte 1 , em que reside aproximadamente um terço da população, o que demonstra quea distribuição destes profissionais contribuiu para melhorar o índice de médicos nos pequenos municípios.

O PMM constitui, então, uma política que vai ao encontro da proposta do SUS, pois amplia a oferta de ações e serviços de saúde que dependem da inclusão desse profissional. No entanto, ainda persistem desafios, como a melhoria das condi- ções de trabalho e infraestrutura dos serviços de saúde, entre outros, que têm dificultado a contratação e a fixação do médico.

Verificou-se que, embora a macrorregião apresente índice de médicos por mil habitantes superior à média nacional, este resultado não revela inequidades identificadas ao se analisar esse índice em nível municipal. Destaca-se que,nos municípios de pequeno porte 1 , a predominância da taxa de médicos foi inferior à média brasileira e, em alguns municípios, inferior a 1 médico por mil habitantes. Esta realidade é oposta ao encontrado nos municípios de médio e grande porte dessa região.

O enfrentamento das desigualdades na distribuição dos médicos nas diferentes regiões do País é complexo, não havendo soluções únicas e isoladas.São necessárias intervenções que articulem a gestão federal, estadual e municipal, estratégias de financiamento, formação dos profissionais de saúde, regulação da formação e do processo de trabalho em saúde epolíticas que fortaleçam as vulnerabilidades dos municípios menores, entre outras ${ }^{31}$. Investir na redução dessa desigualdade é fundamental para garantir a integralidade da atenção, sobretudo em localidades de difícil acesso, como garantia do direito à saúde, especialmente em relação à carência de profissionais no âmbito do SUS.

Finalmente, destaca-se que, apesar da relevância de analisar a distribuição de médicos nos municípios de uma região do estado com base em dados secundários, os resultados deste estudo apontam que estes não são suficientes para elucidar os motivos que levam à desigual distribuição dos médicos nos diferentes municípios, sendo necessário estudos que evidenciem esta realidade.

\section{REFERÊNCIAS}

1. Silveira RP, Pinheiro R. Entendendo a Necessidade de médicos no Interior da Amazônia - Brasil. Rev Bras Educ Méd 2014;38(4):451-459.

2. Póvoa L, Andrade MV. Distribuição geográfica dos médicos no Brasil: uma análise a partir de um modelo de escolha locacional. Cad. Saúde Pública 2006; 22(8):1555-1564.

3. Oliveira FP, Vanni T, Pinto HA, Santos JTR, Figueiredo AM, Araújo SQ, Matos MFM, Cyrino EG. Mais Médicos: um programa brasileiro em uma perspectiva internacional. Interface 2015; 19(54):623-634.

4. Organização Mundial de Saúde. Trabalhadores de Saúde: um perfil global. Relatório Mundial de Saúde; 2006. (Relatório)

5. Scheffer M, Cassenote AJF, Biancarelli A. Demografia Médica no Brasil 2015. Departamento de Medicina Preventiva, Faculdade de Medicina da USP. São Paulo: Conselho 
Regional de Medicina do Estado de São Paulo: Conselho Federal de Medicina, 2015. (Relatório)

6. MachadoMH. Os médicos no Brasil: um retrato da realidade. Rio de Janeiro: Editora FIOCRUZ; 1997.

7. Girardi SN. Identificação de áreas de escassez em recursos humanos em saúde. Observatório de Recursos Humanos em Saúde - Estação de Pesquisa de Sinais de Mercado. Relatório final de atividades. Belo Horizonte; 2012. (Relatório)

8. Silva A. Falta de política de pessoal e distribuição de médicos agravam problemas no SUS. Campinas: Jornal da Unicamp/ Universidade Estadual de Campinas, 2013. (5)

9. Carvalho MS, Souza MF. Como o Brasil tem enfrentado o tema provimento de médicos?. Interface 2013; 17(47):913-926.

10. Maciel Filho R. Estratégias para a distribuição e fixação de médicos em sistemas nacionais de saúde: o caso brasileiro. Rio de Janeiro; 2007. Doutorado [Tese] — Instituto de Medicina Social.

11. Brasil. Instituto Brasileiro de Geografia e Estatísticas-IBGE. Ministério do Planejamento Orçamento e Gestão. Cidades. Estimativa de população. Brasil,2014. [capturado 02 jun. 2014]. Disponível em: http://www.ibge.gov.br/cidadesat/ topwindow.htm?1.

12. Brasil. Cadastro Nacional de Estabelecimentos de Saúde -CNES. Brasil, 2014. [capturado 02 jun. 2014]. Disponível em: http:/ / cnes.datasus.gov.br/.

13. Brasil. Ministério da Saúde. Secretaria de Atenção à Saúde. Portaria n⿳⺈ 134, de 4 de abril de 2011. Dispõe sobre a responsabilidade de atualização do Cadastro Nacional de Estabelecimentos de Saúde pelos Estabelecimentos de Saúde, Municípios, Estados e Distrito Federal. Brasília, 2011. [capturado 02 jun 2015]. Disponível em: http://bvsms.saude. gov.br/bvs/saudelegis/sas/2011/prt0134_04_04_2011. html

14. Brasil. Ministério do Desenvolvimento Social e Combate à Fome e Secretaria Nacional De Assistência Social. Política Nacional de Assistência Social PNAS/ 2004. Brasília: Ministério do desenvolvimento Social, 2004.

15. Região e Redes. Caminhos para a Universalização da Saúde no Brasil. Banco de Indicadores Regionais e Tipologias. Brasil, 2013. [capturado 10 mai. 2015]. Disponível em: http: / / www.resbr.net.br.

16. Brasil. Instituto Brasileiro de Geografia e Estatística-IBGE. Séries Históricas e Estatísticas - Número de Médicos por mil Habitantes. Brasil, 2015. [capturado 12 ago. 2015]. Disponível em: http:/ / seriesestatisticas.ibge.gov.br.

17. Viana, AL et. al. Nota Técnica 1/2015:Quanto o Brasil mudou I - observações a partir da situação das regiões de saúde nos anos 2000 e 2014 - in Novos Caminhos. Região e Redes, 2015.

18. Santos LMP, Costa AM, Girardi SB. Programa Mais Médicos: uma ação efetiva para reduzir iniquidades em saúde. Cien Saude Colet 2015; 20(11):3547-3552

19. Brasil. Ministério da Saúde. Programa Mais Médicos, Governo Federal, 2015. [capturado 02 ago 2015]. Disponível em:http:/ / maismedicos.gov.br/conheca-programa.

20. Girardi SN, Carvalho CL, Araújo JF, Farah JM, Maas LWD, Campos LAB. Índice de escassez de médicos no Brasil: estudo exploratório no âmbito da atenção primária. Brasil, 2016. [capturado 12 fev 2016]. Disponível em: http:/ / www.observatoriorh.org/uruguay/sites/observatoriorh. org.uruguay/files/webfiles/fulltext/publicaciones/indice_de_escassez_de_medicos_no_brasil_estudo_exploratorio_no_ambito_da.pdf.

21. Brasil. Ministério da Saúde. Secretaria de Assistência à Saúde. Departamento de Atenção Básica. Informe da Atenção Básica, nº 21, 2004.

22. Brasil. Ministério da Saúde. A concepção dos pólos como recurso institucional de capacitação, formação e educação permanente de pessoal para saúde da família: Etapa 3. Brasília: Ministério da Saúde, 2002.

23. Campos CV. Por que o médico não fica? Satisfação no Trabalho e Rotatividade dos Médicos do Programa de Saúde da Família do Município de São Paulo. São Paulo; 2005. Mestrado [Dissertação] — Escola de Administração de Empresas de São Paulo da Fundação Getúlio Vargas.

24. Ney MS, Rodrigues PHA. Fatores críticos para a fixação do médico na Estratégia Saúde da Família. Physis 2012; 22(4):1293-1311.

25. Brasil. Instituto Brasileiro de Geografia e Estatística-IBGE. Cidades. Brasil, 2013. [capturado 28 nov. 2016]. Disponível em: http:/ /www.cidades.ibge.gov.br/xtras/temas.php?1ang $=\& \operatorname{codmun}=412270 \&$ idtema $=152 \&$ search $=$ parana $\mid \mathrm{sab}$ audia | produto-interno-bruto-dos-municipios-2013.

26. Rosko MD, Broyles RW. The economics of health care: a reference handbook. New York/London: Greenwood; 1988.

27. Fein R. Studies on physician supply and distribution. Am J Public Health 1954; 44:615-24.

28. Rimlinger GV, Steele HB. An economic interpretation of the spatial distribution of the physicians in the US. South Econ J 1963; 30:1-12.

29. Benham L, Maurizi A, Reder MW. Migration, location and remuneration of medical personnel: physicians and dentists. Rev Econ Stat 1968; 50:332-47.

30. Fleury S, Ouverney ALL. O sistema único de saúde brasileiro - Desafios da gestão em rede. Revista Portuguesa e Brasileira de Gestão 2012; 11:74-83. 
31. Brasil. Ministério da Saúde. Secretaria de Gestão do Trabalho e da Educação na Saúde. Seminário Nacional sobre Escassez, Provimento e Fixação de Profissionais de Saúde em Áreas Remotas de Maior Vulnerabilidade. Brasília: Ministério da Saúde; 2012.

\section{CONTRIBUIÇÃO DOS AUTORES}

Todos os autores participaram da concepção e revisão crítica relevante do conteúdo intelectual do artigo. William Augusto da Fonseca trabalhou na coleta de dados e apresentação dos resultados. Carolina Milena Domingos e Brígida Gimenez Carvalho trabalharam na análise e interpretação dos dados, redação do artigo e aprovação final da versão a ser publicada.

\section{CONFLITO DE INTERESSES}

Não houve conflito de interesses.

ENDEREÇO PARA CORRESPONDÊNCIA

Carolina Milena Domingos

Universidade Estadual de Londrina

Av. Robert Koch, 60

Vila Operária - Londrina

CEP86057-970- PR

E-mail: carolinamdomingos@gmail.com 